Journal of Computer Science 7 (2): 167-172, 2011

ISSN 1549-3636

(C) 2011 Science Publications

\title{
Analysis of Leakage Current to Predict Insulator Flashover Using Artificial Neural Network
}

\author{
${ }^{1}$ N. Narmadhai and ${ }^{2}$ A. Ebenezer Jeyakumar \\ ${ }^{1}$ Department of Electrical and Electronics Engineering, \\ Government College of Technology, Coimbatore, India \\ ${ }^{2}$ Director (Academics) Sri Ramakrishna Engineering College, \\ Coimbatore, India
}

\begin{abstract}
Problem statement: The phenomenon of flashover in polluted insulators has been continued by the study of the characteristics of contaminating layers deposited on the surface of insulators in high voltage laboratories. In the literature, Experimental investigations have been carried out on a real insulator or a flat plate model of insulators under high voltage application. This study proposed the Equivalent insulator flat plate model for studying the flashover phenomena due to pollution under wet conditions even at low voltage. Laboratory based tests were carried out on the model under AC voltage at different pollution levels. Different concentrations of salt solution has been prepared using sodium chloride, Kaolin and distilled water representing the various contaminations. Leakage current during the experimental studies were measured for various polluted conditions. Approach: A new model of $\mathrm{Vc}=\mathrm{f}\left(\mathrm{V}, \mathrm{I}_{\text {initial }}, \mathrm{I}_{\mathrm{em}}, \mathrm{I}_{\mathrm{emax}}\right.$ and $\left.\mathrm{I}_{\sigma}\right)$ based on artificial neural network has been developed to predict flashover from the analysis of leakage current. The input variable to the artificial neural network are mean $\left(\mathrm{I}_{\text {mean }}\right)$, Maximum(Imax) and standard deviation $\left(\mathrm{I}_{\sigma}\right)$ of leakage current extracted along with the initial value of leakage current $\mathrm{I}_{\text {initial }}$ and the input voltage(V). The target obtained was used to evaluate the performance of the neural network model. Results: The optimum process has been carried out based on the training accuracy measured by RMSE, the network converged to a threshold of 0.0001.The trained model prediction is in good agreement with the actual results and the $\mathrm{R}^{2}$ value of the developed model is 0.99996. Conclusion: The developed ANN model is well-suited for the analysis of leakage current to predict flashover on the insulator surface with high accuracy.
\end{abstract}

Key words: Flashover voltage, insulator model, leakage current, artificial neural network, Equivalent Salt Deposit Density (ESDD), Non-Soluble Deposit Density (NSDD), Backpr Opagation (BP), Root-Meansquare (RMS), various contamination

\section{INTRODUCTION}

Insulators used in outdoor electric power transmission lines are exposed to outdoor environmental contaminations. Contamination on outdoor insulators enhances the chances of flashover. Depending on the nature and duration of exposure, deposits of wind-carried industrial, sea and dust contaminants build up on the insulator surface as a dry layer. The leakage current path through a layer of dry contaminants on an insulator surface is capacitive wherein the current amplitude is small and sinusoidal. The dry contaminant layer becomes conductive when exposed to light rain or morning dews. As wetting progresses, the leakage current path changes from capacitive to resistive with simultaneous increase in current amplitudes. The increase in leakage current dries the conducting layer and forms the dry bands around the areas with high current density. These dry bands interrupt the current flow and most of the applied voltages are impressed across these narrow dry bands. If the dry bands cannot withstand the voltage, localized arcing develops and the dry bands will be spanned by discharges. The arcs merge together and form a single arc, which triggers the surface flashover (Jeyakumar, 1991).

The contamination severity determines the frequency and intensity of arcing and, thus the probability of flashover. In favorable conditions when the level of contamination is low, layer resistance is high and arcing continues until the sun or wind dries the layer and stops the arcing. Continuous arcing is

Corresponding Author: N. Narmadhai, Department of Electrical and Electronics Engineering,

Government College of Technology, Coimbatore, India 
harmless for ceramic insulators. The mechanism described above shows that heavy contamination and wetting may cause insulator flashover and service interruptions. Contamination in dry conditions is harmless. Hampton investigated the voltage distribution along the wet, polluted surface of a flat insulating strip and the method of dry band formation, with subsequent growth of discharges on the polluted surface (Hampton, 1964). Karady measured the peak leakage current and correlated the current with the flashover voltage. He suggested that the flashover is imminent if the leakage current peak exceeds $100 \mathrm{~mA}$ (Karady et al., 1999). In practice, there are various contaminant types that settle on outdoor insulators. These contaminants can be classified as soluble and insoluble. Insulators located near coastal regions are typically contaminated by soluble contaminants, especially salt (or sodium chloride). Insulators located near cement or study industries are typically contaminated by non-soluble contaminants such as calcium chloride, carbon and cement dust. Irrespective of the type of contaminant, flashover can occur as long as the salts in the contaminant are soluble enough to form a conducting layer on the insulator's surface. In order to quantify the contaminants on the surface of the insulators, the soluble contaminants are expressed in terms of Equivalent Salt Deposit Density (ESDD), which correlates to $\mathrm{mg}$ of $\mathrm{NaCl}$ per unit surface area. Nonsoluble contaminants are expressed in terms of Non-Soluble Deposit Density (NSDD), which correlates to mg of kaolin per unit surface area.

Many researchers studied that the leakage current due to the contamination level is the main cause for flashover. Farag, (1995) applied ANN as function estimators in the insulator flashover studies. The training and the test data of ANN are obtained from the experimental studies carried out on a flat plate model of polluted insulator under power-frequency voltage. The combinations of ANN parameters which give best result are clearly identified and the model results are compared with the experimental results. In Guan Zhicheng and Guoshun (1994) the maximum value of leakage current has the definite relationship with the flashover voltage of the polluted insulator used to express the pollution degree of insulator. Artificial neural networks can be used in problems requiring function approximation, modeling, pattern recognition and classification, estimation and prediction (Haykins, 1998, Limsombunchai and Minso, 2004; Moussaoui et al., 2006).

Li et al. (2010) extracted three characteristics of the leakage current, namely the mean value, maximum value and the standard deviation of the RootMeansquare (RMS) value of the leakage current, from the recorded value. They describe jointly the current contamination levels of an insulator surface. In addition, regression equations between the three characteristics and various contamination levels have been established. The same three characteristics have been selected and used as the inputs of neural network model.

\section{MATERIALS AND METHODS}

Equivalent insulator model: The simplified geometrical models equivalent to actual insulator is being widely used for the purpose of flashover analysis. Among these models, the basic flat trough model has merited extensive attention in the context of pollution flashover. So the proposed model, equivalent to standard disc insulator made of an insulating glass material with two copper terminals, one on cap and another at the pin.

Experimental setup: A proposed equivalent insulator trough model (Jeyakumar, 1991) of dimension $18.5 \times 0.6 \times 0.2 \mathrm{~cm}$ is used for the contamination flashover experiments(Narmadhai et al., 2010). The principal application of this equivalent model would be to help simulate as much as possible the practical conditions of high voltage insulators in the application of low voltage itself. In artificial testing, a contaminant is usually substituted by a dissolved mixture of an inert binder-Kaolin and $\mathrm{NaCl}$ salt. The inert binder is supposedly non-conducting and the quantity of salt represents the level of contamination. Contamination salt solution was prepared for various $\mathrm{NaCl}$ values of $15,20,25$ and $30 \mathrm{~g}$. The mixture, usually dissolved in distilled water is known as slurry which is thoroughly mixed as per IEC standard (British Standards Institute Staff, 1993). Before coating, the trough is initially washed and wiped clean and dry. The experimental setup to measure the leakage current is shown in Fig. 1. The slurry is poured so that it rolls off uniformly in the trough.

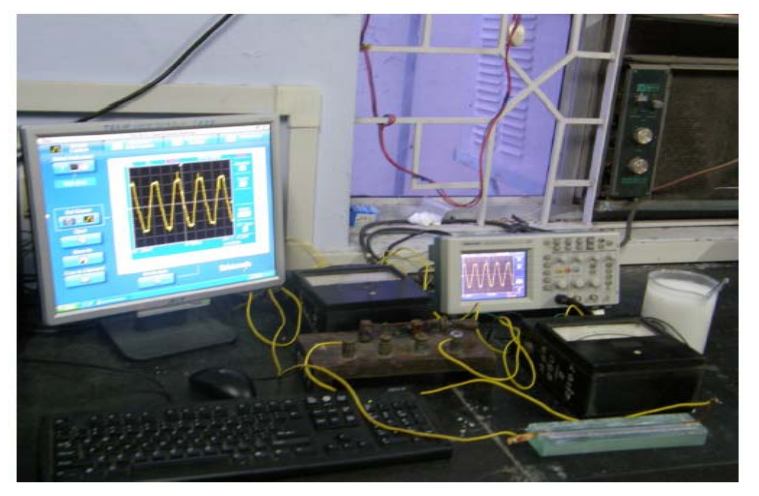

Fig. 1: Experimental setup 
A test voltage of $230 \mathrm{~V}, 50 \mathrm{~Hz}$ was applied across the terminals and the leakage current is monitored through the suitable measuring meter from the instant of application of voltage till the formation of dry band. The dry band was precisely located on the model. Its shape, contour of growth and locations were physically measured. The test results either in a flashover or a withstand. The leakage current is measured using ammeter and also captured in Digital Signal Oscilloscope. The captured leakage current waveform is stored for further analysis.

Neural network model for predicting flashover based on the characteristics of leakage current:

Neural network: ANN is a computer model representing the biological brain. It consists of a set of interconnected simple processing units (neurons or nodes) bonded with weight connections which combine to output a signal to solve a certain problem based on the input signals it received. The process of training an ANN can be supervised and unsupervised learning. In supervised learning, the ANN is previously trained and the target is fixed for a particular task. The ANN are adjusted or trained, so that a particular input leads to a specific target output. In the output layer the data is compared with the predefined target and the error is calculated. This error is propagated back and the weight updation is done. The process is repeated to meet the minimum error value, the training task is deemed complete. In unsupervised learning, the target is not fixed; weights are adjusted autonomously until a balanced condition is reached when the weights do not change further.

Backpropagation (BP) is the most widely used supervised learning algorithm because of its simplicity.

Characteristics of leakage current: The input parameters selected for the artificial neural network for flashover prediction were the three characteristics extracted from the measured leakage current are mean value of leakage current $\left(\mathrm{I}_{\mathrm{em}}\right)$, Maximum value of the leakage current $\left(\mathrm{I}_{\mathrm{emax}}\right)$ and Standard deviation of leakage current $\left(I_{\sigma}\right)$ along with the initial value of leakage current ( $\left.\mathrm{I}_{\text {initial }}\right)$ and the input supply voltage. The three characteristics, (Li et al., 2010) i.e., the mean value, maximum value and standard deviation of the leakage current, are proposed as follows:

$$
\begin{aligned}
& I_{e m}=\left(\sum_{1}^{N} \mathrm{Ie}(\mathrm{i})\right) / \mathrm{N} \\
& \mathrm{I}_{\mathrm{em}}=\max \left(\mathrm{I}_{\mathrm{e}}(\mathrm{i})\right) \\
& \mathrm{I}_{\sigma}=\sqrt{\left(\sum_{1}^{\mathrm{N}} \mathrm{Ie}(\mathrm{i})-\mathrm{I}_{\mathrm{em}}\right)^{2} / \mathrm{N}}
\end{aligned}
$$

Where:

$\mathrm{N}=$ The total number of sampling points in the test time

$\mathrm{I}_{\mathrm{e}}(\mathrm{i})=$ The leakage current value in one sampling period

$\mathrm{I}_{\mathrm{em}}=$ The mean value of leakage current in the test time

$I_{e m a x}=$ The maximum value of leakage current in the test time

$I_{\sigma}=$ The standard deviation of leakage current in the test time

The standard deviation represents the degree of deviation between each sampling value and the mean value and also means the discrete distribution degree among all sampling points during the test time.

Normalization of input variables: Normalization is a transformation performed on a single data input to distribute the data evenly and scale it into an acceptable range for the neural network. Such preprocessing of data ensures a uniform statistical distribution of each input value. If the input and the output variables are not of the same order of magnitude, some variables may appear to have more significance than they actually do. Input and output data can be normalized in many ways. In this work, the minimum and maximum values of a group of data are used to normalize each single input using Eq. 4 given below:

$\mathrm{I}=\frac{\text { Im easured }}{\mathrm{I}_{\mathrm{mac}}+\mathrm{I}_{\mathrm{min}}}$

where, $I_{\max }$ and $I_{\min }$ are maximum and minimum values of measured input data. The range of normalized values of input data ranges from 0.2-0.8.

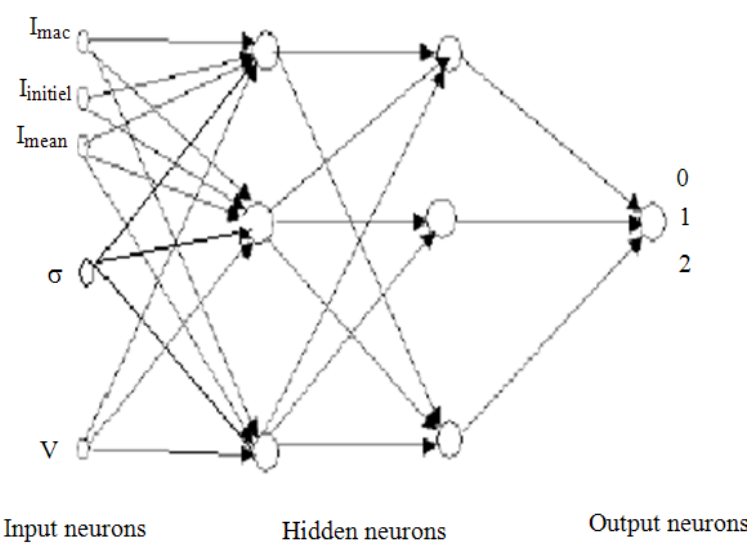

Fig. 2: Neural network Model 
Training of ANN for flashover prediction: The multilayer feed forward neural network model with back propagation algorithm for training shown in Fig. 2, illustrates our implemented neural network. For training the neural network to predict flashover, Five input neurons are used, three to indicate the leakage current characteristics, (the mean value, maximum value and standard deviation) and two input neurons to indicate the input voltage and initial value of the leakage current in the input layer, three neurons in the hidden layers and one neuron in the output layer. The hidden layer is using tangent sigmoid transfer function and output neuron is using linear transfer function. All the input data used in the proposed neural networks were actual collected data based on actual measurements. The normalized training patterns are fed to the model. Leven-Marquardt algorithm is used for training as it converge fast and the function 'trainlm' is invoked .Using trial and error the numbers of nodes in the hidden layer are determined.

The optimization process has been carried out based on MSE and $R^{2}$ value by varying the number of hidden layers. The number of hidden layer neurons is varied from 1-15. It was found that best $\mathrm{R}^{2}$ and MSE is obtained for 3 nodes in the hidden layer. The values of $\mathrm{R}^{2}$ and MSE for various hidden neuron number is given in Table 1.

Table 2 compares the effect of number of hidden layers on the convergence rate of the training process. Finally, the ANN network with three hidden layers has better effect on the convergence based on the

Table 1: R2 and MSE value for various number of hidden layers

\begin{tabular}{lrl}
\multicolumn{3}{l}{ Table 1: R2 and MSE value for various number of hidden layers } \\
\hline No. of nodes in hidden layers & \multicolumn{1}{l}{ MSE } & $\mathrm{R}^{2}$ \\
\hline 1 & 3.220 & 0.95400 \\
2 & 3.910 & 0.91715 \\
3 & 0.640 & 0.99999 \\
4 & 2.500 & 0.99994 \\
5 & 5.480 & 0.99994 \\
6 & 0.660 & 0.99996 \\
7 & 1.210 & 0.99998 \\
8 & 4.100 & 0.99990 \\
9 & 4.970 & 0.99992 \\
10 & 0.849 & 0.99986 \\
11 & 14.000 & 0.99994 \\
12 & 3.250 & 0.99988 \\
13 & 12.300 & 0.99986 \\
14 & 0.955 & 0.99999 \\
15 & 8.500 & 0.99996 \\
\hline
\end{tabular}

Table 2: Effect of numbers of hidden layers

\begin{tabular}{llccc}
\hline $\begin{array}{l}\text { Number of } \\
\text { hidden layers }\end{array}$ & $\begin{array}{l}\text { Number of nodes } \\
\text { in hidden layers }\end{array}$ & MSE & RMSE & $\mathrm{R}^{2}$ \\
\hline 1 & 3 & 0.640 & 0.00538 & 0.99994 \\
2 & $3 / 3$ & 6.850 & 0.05540 & 0.99986 \\
2 & $3 / 8$ & 2.700 & 0.01250 & 0.99998 \\
3 & $3 / 8 / 8$ & 0.831 & 0.00591 & 0.99998 \\
3 & $3 / 8 / 11$ & 0.617 & 0.00367 & 0.99999 \\
4 & $3 / 8 / 11 / 11$ & 2.540 & 0.00365 & 0.99988 \\
\hline
\end{tabular}

minimum RMSE than the single hidden layer. The test output results are calculated using three hidden layer with three neurons in first hidden layer, eight neurons in second hidden layer and eleven neurons in third hidden layer.

\section{RESULTS}

The training is carried out for 300 set of input data and the neural network is tested for 9 set of input data to attain the performance goal of 0.0001.The learning rate and momentum factors are chosen as 0.9 and 0.3 and maximum of 500 epochs are considered. The goal is achieved in 79epochswith error in the range of 0.0001 . The target for no arcing and flashover are trained to be 0 , Initialization of arc and no flashover are trained to be 1 and for severe arcing and flashover, it is trained to be 2 .

From the trained network, testing is carried out and test results are compared with experimental results and the error difference are shown in Table 3. The overall testing accuracy equals to $99.73 \%$. with $99.88 \%$ accuracy for no flashover, $99.91 \%$ for flashover case and $99.41 \%$ for forecasting i.e arcing and no flashover.

\section{DISCUSSION}

The performance graph of the variations of mean square error in the training process is shown in Fig. 3 against the number of iterations.

The occurrence of flashover i.e., the target noted from the measured leakage current during the experiment and the simulated ANN result have been plotted for nine tests chosen randomly from the data stored are as shown in Fig. 4. The error difference is found to be very less than 0.007 .

The correlation coefficient ranges between [-1, 1].The goal is to have the value of $R$ close to 1 as possible. The correlation between real and estimated targets for this case is shown in Fig. 5. As it is shown, the correlation is $\mathrm{R}^{2}=0.99996$. It must be mentioned that the ideal value for the correlation is 1 , so 0.99996 is definitely an acceptable value.

Table3: Comparison between Test and Simulation results

\begin{tabular}{llll}
\hline & \multicolumn{3}{c}{ Simulated result } \\
Occurrence of flashover & Target (ANN) & Error difference \\
\hline No arc and flashover & 0 & 0.0017 & 0.0017 \\
& 0 & 0.0002 & 0.0002 \\
Initialization of arcing & 0 & 0.0017 & 0.0017 \\
and no flashover & 1 & 0.9927 & 0.0073 \\
Severe arcing and flashover & 1 & 0.9933 & 0.0067 \\
& 1 & 0.9963 & 0.0037 \\
& 2 & 1.9992 & 0.0008 \\
& 2 & 1.9983 & 0.0001 \\
\hline
\end{tabular}




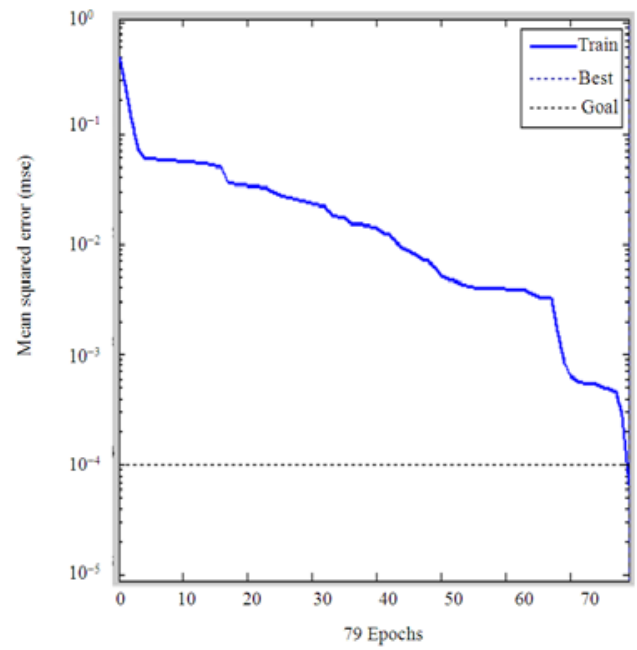

Fig. 3: Performance graph

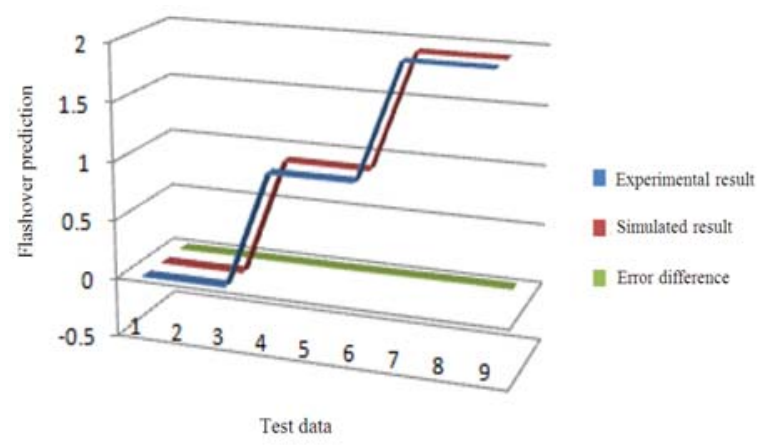

Fig. 4: Comparison between experimental result and simulated ANN

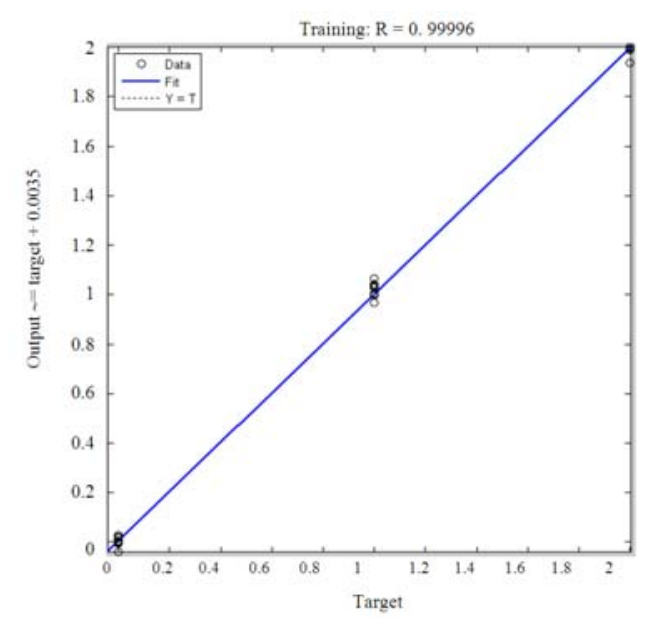

Fig. 5: Correlation between real and estimated values of flashover

\section{CONCLUSION}

In this study, a simple scheme is proposed to predict flashover of equivalent insulator model using ANN. To simplify the mathematical analysis, tests were done on a flat trough model of simple geometry. The maximum leakage current is determined. The laboratory model test results either in a flashover or a withstand. The network was modeled as $\mathrm{Vc}=\mathrm{f}(\mathrm{V}$, $I_{\text {initial }} I_{e m}, I_{e m a x}$ and $I \sigma$ ) to predict the flashover accurately to assess the condition of the insulation system. The high $R^{2}$ value indicates a high generalization capability of the developed model. From the comparison, the simulated ANN result with the measured leakage current data distinguishes the occurrence of flashover and gives indication which is considered to be very fast. The proposed technique shows an effective solution against the occurrence of a pollution flashover and indicates the need for cleaning of the insulators.

\section{REFERENCES}

British Standards Institute Staff, 1993. Artificial pollution Tests on High-Voltage Insulators to be Used on A. C. Systems. 1st Edn., B S I Standards, Switzerland, ISBN: 0580225216, pp: 40.

Farag, A.S., 1995. Estimation of Polluted insulators flashover time using artificial neural networks. Proceeding of the International IEEE/IAS Conference on Industrial Automation and Control: Emerging Technologies, May 22-27, Taipei, Taiwan, pp: 320-327. DOI: 10.1109/IACET.1995.527582

Hampton, B.F., 1964. Flashover mechanism of polluted insulation. Elec. Power, 10: 113-113. DOI: 10.1049/ep.1964.0112

Haykins, S.S., 1998. Neural Networks A Comprehensive Foundation. 2nd Edn., Prentice Hall, USA., ISBN: 0132733501, pp: 842.

Jeyakumar, A.E., 1991. Development of verisimilar juxtaposition model and study of physical phenomena on polluted insulators. Ph.D Thesis, Department of Electrical Engineering, Anna University Madras, India. http://www.annauniv.edu/Library/thesispage11.html

Karady, G., F. Amarh and R. Sundararajan, 1999. Dynamic modeling of ac insulator flashover characteristics. Proceeding of the 11th International Symposium of High Voltage Engineering, Aug. 23-27, Arizona State University, USA., pp: 107110. DOI: $10.1049 /$ cp:19990804

Li, J., C. Sun,W. Sima, Q. Yang and J. Hu, 2010. Contamination level prediction of insulators based on the characteristics of leakage current. IEEE Trans. Power Deliv., 25: 417-424. DOI: 10.1109/TPWRD.2009.2035426 
Limsombunchai, V., C. Gan and Minso, 2004. House price prediction: hedonic price model Vs. artificial neural network. Am. J. Applied Sci., 1: 193-201. DOI: 10.3844/ajassp.2004.193.201

Moussaoui, A., Y. Selaimia and H.A. Abbassi, 2006. Hybrid hot strip rolling force prediction using a Bayesian trained artificial neural network and analytical models. Am. J. Applied Sci., 3: 1885-1889. DOI: 10.3844/ajassp.2006.1885.1889

Narmadhai, N., S. Suresh and A.E. Jeyakumar, 2010. Laboratory study of leakage current and measurement of ESDD of equivalent insulator flat model under various polluted conditions. Int. J. Comput. Sci. Inform. Security, 8: 157-161.
Zhicheng, G. and C. Guoshun, 1994. A study on the leakage current along the surface of polluted insulator. Proceeding of the 4th International Conference on Properties and Applications of Dielectric Materials, July 3-8, Brisbane, Qld., Australia, pp: 495-498. DOI: 10.1109/ICPADM.1994.414055 